\title{
Single-spin polaron memory effect in quantum dots and single molecules
}

\author{
Dmitry A. Ryndyk, Pino D’Amico, and Klaus Richter \\ Institute for Theoretical Physics, University of Regensburg, D-93040 Regensburg, Germany
}

(Received 25 September 2009; published 31 March 2010)

\begin{abstract}
We propose theoretically a spin memory effect in quantum dots and single molecules with strong electronvibron interaction and coupled to ferromagnetic leads. The controlled electrical switching between spin states is achieved due to an interplay between Franck-Condon blockade of electron transport at low voltages and spin-dependent tunneling at high voltages. Spin lifetimes, currents, and spin polarizations are calculated as a function of the bias voltage by the master-equation method. We further propose to use a third ferromagnetic tunneling contact to probe and readout the spin state.
\end{abstract}

DOI: 10.1103/PhysRevB.81.115333

PACS number(s): 85.65.+h, 72.25.-b, 73.23.-b, 85.75.-d

\section{INTRODUCTION}

One of the most promising directions in the fields of molecular electronics and spintronics is the experimental and theoretical investigation of spin manipulation in quantum dots and single molecules. In particular, new methods have been recently developed to investigate spin states of single atoms and molecules, e.g., using spin-polarized scanningtunneling spectroscopy. ${ }^{1,2}$ Motivated by such achievements the promising question arises whether a single-spin memory effect (namely, the controlled switching of the spin state of a single electron by applying an external voltage to a system) is possible. The main challenge is to combine long spin lifetimes and fast switching.

One of the ways for single-spin manipulation is based on the interplay of charge, spin, and vibron degrees of freedom in molecular junctions. In various experiments signatures of the electron-vibron (e-v) interaction have been observed in atomic-scale structures. ${ }^{3-5}$ In the case of strong e-v interaction the formation of a local polaron can lead to a chargememory effect, which was first predicted long time ago, ${ }^{6}$ and has been recently reconsidered theoretically in more detail: $^{7-12}$ neutral and charged (polaron) states correspond to different local minima of an effective energy surface and are metastable if the e-v interaction is strong enough. By applying an external voltage, one can change the charge state of this bistable system, an effect that is accompanied by hysteretic charge-voltage and current-voltage curves. A similar memory effect was found in recent scanning tunnel microscope experiments ${ }^{13}$ as a multistability of neutral and charged states of single metallic atoms coupled to a metallic substrate through a thin insulating ionic film; the corresponding single-level polaron model was discussed in Refs. 11 and 12.

In this paper we propose an approach to observe a singlespin memory effect by combining the polaron-memory mechanism and spin-dependent tunneling. To this end we consider a single-level and single-vibron quantum system between magnetic leads (Fig. 1). We study the case of a symmetric junction with antiparallel magnetizations of left and right leads; besides a third electrode can be used as a gate or to probe the spin state. In the case of parallel orientation of magnetization in the leads similar effects are observed but spin-up and spin-down states are not equivalent and the memory effect is hidden. The problem can be solved with well-controlled approximation in the limit of weak coupling to the leads, where the master equation for sequential tunneling can be used. Thus we focus our major discussion on this limit.

This paper is organized as follows: in Sec. II we introduce the electron-vibron model and describe the master equation method used for calculations. In Sec. III we consider spin lifetimes and show the existence of metastable spin states. In the Sec. IV bias-voltage-controlled switching between memory states is discussed and we conclude in Sec. V.

\section{MODEL AND METHOD}

The Hamiltonian of the single-level polaron (AndersonHolstein) model is

$$
\begin{aligned}
\hat{H}= & \sum_{\sigma} \tilde{\epsilon}_{\sigma} d_{\sigma}^{\dagger} d_{\sigma}+\omega_{0} a^{\dagger} a+\lambda\left(a^{\dagger}+a\right) \hat{n}+U \hat{n}_{\uparrow} \hat{n}_{\downarrow} \\
& +\sum_{i k \sigma}\left[\left(\epsilon_{i k \sigma}+e \varphi_{i}\right) c_{i k \sigma}^{\dagger} c_{i k \sigma}+\left(V_{i k \sigma} c_{i k \sigma}^{\dagger} d_{\sigma}+\text { H.c. }\right)\right] .
\end{aligned}
$$

Here the first line describes the free electron states with energies $\tilde{\boldsymbol{\epsilon}}_{\sigma}$, the free vibron of frequency $\omega_{0}$, and the electron-vibron and Coulomb interactions with coupling strength $\lambda$ and $U$, respectively; $\sigma$ is the spin index, and $\hat{n}_{\sigma}=d_{\sigma}^{\dagger} d_{\sigma}, \hat{n}=\hat{n}_{\uparrow}+\hat{n}_{\downarrow}$. The other terms in Eq. (1) are the Hamiltonians of the leads and the tunneling coupling ( $i=L, R$ is the lead index, $k$ labels the electronic states). The bias voltage $V$ is introduced through the left and right elec-

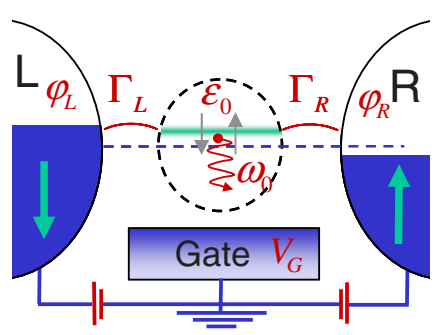

FIG. 1. (Color online) Schematic of the considered system: a gated single-level quantum dot interacting with a vibron and coupled to ferromagnetic leads. The gate electrode can be considered also as a third test electrode coupled weakly to the system. 
trical potentials, $V=\varphi_{L}-\varphi_{R}$. The energy $\tilde{\epsilon}_{\sigma}=\epsilon_{\sigma}+e \varphi_{0}$ includes the bare level energies $\epsilon_{\uparrow}, \epsilon_{\downarrow}$ and the electrical potential $\varphi_{0}$ describing the shift of the central level by the gate voltage $V_{G}$ and by the bias voltage drop between the left and right lead: $\varphi_{0}=\varphi_{R}+\eta\left(\varphi_{L}-\varphi_{R}\right)+\alpha V_{G}$, where $0<\eta<1$ describes the symmetry of the voltage drop across the junction, $\eta=0.5$ stands for the symmetric case considered below. We assume the simplest linear dependence of the molecular potential $\left(\eta=\right.$ const) but its nonlinear dependence ${ }^{14,15}$ can be easily included in our model. We further choose $\epsilon_{\uparrow}=\epsilon_{\downarrow}=\epsilon_{0}$ and as reference energy $\epsilon_{0}=0$ for $V_{G}=0$ and assume a linear capacitive coupling, $\epsilon_{0}=\alpha e V_{G}$, putting $\alpha=1$.

The coupling to the leads is incorporated in the levelwidth function

$$
\Gamma_{i \sigma}(\epsilon)=2 \pi \sum_{k}\left|V_{i k \sigma}\right|^{2} \delta\left(\epsilon-\epsilon_{i k \sigma}\right) .
$$

The spin-dependent densities of states in the leads and the tunneling matrix elements are assumed to be energyindependent (wide-band limit) so that $\Gamma_{L \sigma}$ and $\Gamma_{R \sigma}$ are constants. The full level broadening is $\Gamma_{\sigma}=\Gamma_{L \sigma}+\Gamma_{R \sigma}$. Below we consider a symmetric junction with antiparallel magnetization of the leads and use the notation $\Gamma_{L \downarrow}=\Gamma_{R \uparrow}=\Gamma$ for majority spins and $\Gamma_{L \uparrow}=\Gamma_{R \downarrow}=\kappa \Gamma$ for minority spins, $\kappa \ll 1$.

The spin effects addressed are particularly pronounced in the limit of large $U$, i.e., we neglect the doubly occupied state so that only three states in the charge sector should be considered: neutral $|0\rangle$, charged spin up $|\uparrow\rangle$ and charged spin down $|\downarrow\rangle$. Employing the polaron canonical transformation, ${ }^{16,17}$ the corresponding eigenstates of the isolated system $(\Gamma=0)$ read

$$
\begin{gathered}
\left|\psi_{0 q}\right\rangle=\frac{\left(a^{\dagger}\right)^{q}}{\sqrt{q !}}|0\rangle, \\
\left|\psi_{\sigma q}\right\rangle=e^{-\lambda / \omega_{0}\left(a^{\dagger}-a\right) d_{\sigma}^{\dagger} d_{\sigma}} d_{\sigma}^{\dagger} \frac{\left(a^{\dagger}\right)^{q}}{\sqrt{q !}}|0\rangle
\end{gathered}
$$

with the eigenenergies

$$
E_{0 q}=\omega_{0} q, \quad E_{\sigma q}=\widetilde{\epsilon}_{\sigma}^{\prime}+\omega_{0} q, \quad \widetilde{\epsilon}_{\sigma}^{\prime}=\tilde{\epsilon}_{\sigma}-\frac{\lambda^{2}}{\omega_{0}} .
$$

Here the quantum number $q$ characterizes vibronic eigenstates, which are superpositions of states with different number of bare vibrons.

Accounting for all possible single-electron tunneling processes for both leads, the incoming and outgoing tunneling rates read

$$
\begin{aligned}
\Gamma_{q q^{\prime}}^{\sigma 0} & =\sum_{i=L, R} \Gamma_{i q q^{\prime}}^{\sigma 0}=\sum_{i=L, R} \Gamma_{i \sigma}\left|M_{q q^{\prime}}\right|^{2} f_{i}^{0}\left(E_{\sigma q}-E_{0 q^{\prime}}\right) \\
& =\sum_{i=L, R} \Gamma_{i \sigma}\left|M_{q q^{\prime}}\right|^{2} f_{i}^{0}\left[\widetilde{\epsilon}_{\sigma}^{\prime}+\omega_{0}\left(q-q^{\prime}\right)\right],
\end{aligned}
$$

$$
\begin{aligned}
\Gamma_{q q^{\prime}}^{0 \sigma} & =\sum_{i=L, R} \Gamma_{i q q^{\prime}}^{0 \sigma}=\sum_{i=L, R} \Gamma_{i \sigma}\left|M_{q q^{\prime}}\right|^{2}\left[1-f_{i}^{0}\left(E_{\sigma q^{\prime}}-E_{0 q}\right)\right] \\
& =\sum_{i=L, R} \Gamma_{i \sigma}\left|M_{q q^{\prime}}\right|^{2}\left\{1-f_{i}^{0}\left[\widetilde{\boldsymbol{\epsilon}}_{\sigma}^{\prime}-\omega_{0}\left(q-q^{\prime}\right)\right]\right\} .
\end{aligned}
$$

Here $f_{i}^{0}(\epsilon)$ is the equilibrium Fermi function in lead $i$ shifted by the external potential, $f_{i}^{0}(\epsilon)=f^{0}\left(\epsilon-e \varphi_{i}\right)$, and $M_{q q^{\prime}}$ is the Franck-Condon matrix element

$$
M_{q q^{\prime}}=\left\langle 0\left|\frac{a^{q}}{\sqrt{q !}} \exp \left[\frac{\lambda}{\omega_{0}}\left(a^{\dagger}-a\right)\right] \frac{\left(a^{\dagger}\right)^{q^{\prime}}}{\sqrt{q^{\prime} !}}\right| 0\right\rangle .
$$

It is symmetric in $q-q^{\prime}$ and can be calculated analytically (see Refs. 18-22 for details). For $q<q^{\prime}$ it reads

$$
M_{q<q^{\prime}}=\sum_{l=0}^{q} \frac{(-g)^{l} \sqrt{q ! q^{\prime} !} e^{-g / 2} g^{\left(q^{\prime}-q\right) / 2}}{l !(q-l) !\left(l+q^{\prime}-q\right) !},
$$

where $g=\left(\lambda / \omega_{0}\right)^{2}$ is the Huang-Rhys factor. ${ }^{23}$

In Eq. (6) the incoming rate $\Gamma_{q q^{\prime}}^{\sigma 0}$ describes tunneling of one electron with spin $\sigma$ from the lead to the dot changing the state of the dot from $\left|0 q^{\prime}\right\rangle$ to $|\sigma q\rangle$. The outgoing rate $\Gamma_{q q^{\prime}}^{0 \sigma}$ corresponds to the transition from $\left|\sigma q^{\prime}\right\rangle$ to $|0 q\rangle$.

In the sequential tunneling regime the master equation for the probability $P_{q}^{n}(t), n=0, \uparrow, \downarrow$, to find the system in one of the polaron eigenstates, Eqs. (3) and (4), can be written $a^{18-22}$ (see also the master equation approach for a quantum dot between ferromagnetic leads in Refs. 24 and 25, and references therein)

$$
\frac{d P_{q}^{n}}{d t}=\sum_{n^{\prime} q^{\prime}} \Gamma_{q q^{\prime}}^{n n^{\prime}} P_{q^{\prime}}^{n^{\prime}}-\sum_{n^{\prime} q^{\prime}} \Gamma_{q^{\prime} q}^{n^{\prime} n} P_{q}^{n}+I^{V}[P] .
$$

Here the first term represents the tunneling transition into the state $|n q\rangle$ and the second term the transition out of the state $|n q\rangle . I^{V}[P]$ is the vibron scattering integral describing the relaxation of the vibrons to the thermal equilibrium.

Finally, the average charge and the spin polarization are

$$
Q=e \sum_{q}\left(P_{q}^{\uparrow}+P_{q}^{\downarrow}\right), \quad S=\sum_{q}\left(P_{q}^{\uparrow}-P_{q}^{\downarrow}\right),
$$

respectively, and the average current (from the left or right lead) reads

$$
J_{i=L, R}=e \sum_{\sigma q q^{\prime}}\left(\Gamma_{i q q^{\prime}}^{\sigma 0} P_{q^{\prime}}^{0}-\Gamma_{i q q^{\prime}}^{0 \sigma} P_{q^{\prime}}^{\sigma}\right)
$$

with $\Gamma_{i q q^{\prime}}^{\sigma 0}$ and $\Gamma_{i q q^{\prime}}^{0 \sigma}$ defined in Eqs. (6) and (7).

\section{SPIN LIFETIMES}

Let us first calculate the characteristic lifetimes of the neutral, spin-up, and spin-down states being in the vibronic ground state $q=0$. We define the switching rates $\gamma^{\sigma 0}$ from the neutral to the charged state with spin $\sigma$ and vice-versa as the sum of the rates of all possible processes which change these states 


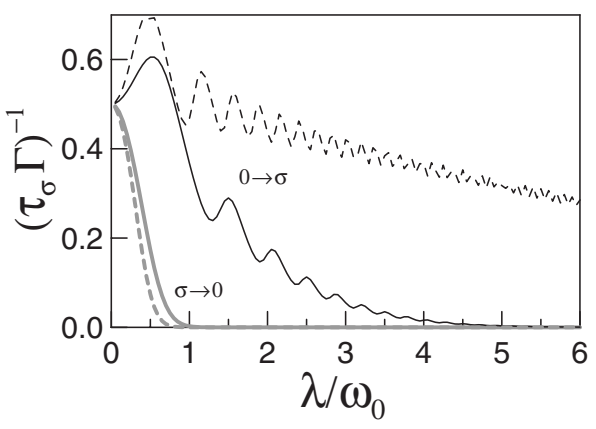

FIG. 2. Inverse lifetime $\gamma^{\sigma 0} / \Gamma$ of the neutral state (thin black lines) and the inverse spin lifetime $\left(\tau_{\sigma} \Gamma\right)^{-1}$ (thick gray lines) as a function of the scaled electron-vibron coupling $\sqrt{g}=\lambda / \omega_{0}$ at $\epsilon_{0}=\lambda^{2} / 2 \omega_{0}$ (solid lines) and at $\epsilon_{0}=0.1 \lambda^{2} / \omega_{0}$ (dashed lines), $T=0.1 \omega_{0}$.

$$
\gamma^{\sigma 0}=\sum_{q} \Gamma_{q 0}^{\sigma 0}, \quad \gamma^{0 \sigma}=\sum_{q} \Gamma_{q 0}^{0 \sigma}
$$

In the sequential tunneling approximation the spin lifetime $\tau_{\sigma}$ is determined by the switching rate from the charged state $\gamma^{0 \sigma}$ through Eqs. (7) and (13). It reads (setting the Fermi level to zero) ${ }^{11}$

$$
\tau_{\sigma}^{-1}=\gamma^{0 \sigma}=(1+\kappa) \Gamma \sum_{q} \frac{e^{-g} g^{q}}{q !} f^{0}\left(-\widetilde{\epsilon}_{\sigma}^{\prime}+\omega_{0} q\right) .
$$

At large $g$ the sequential tunneling rates are exponentially suppressed (Franck-Condon blockade) and the cotunneling contribution to $\tau_{\sigma}^{-1}$ may become dominant. It is particularly important for the spin lifetime because cotunneling does not change charge but may change spin. The cotunneling rate can be estimated as ${ }^{22}$

$$
\tau_{\sigma}^{-1(c t)} \approx \frac{\kappa \Gamma^{2} T \omega_{0}^{2}}{\lambda^{4}}
$$

Although the cotunneling contribution is not suppressed exponentially, it is of second order in the tunneling coupling and suppressed additionally by the small polarization parameter $\kappa$ and large $\lambda$. At typical parameters, considered in this paper (weak coupling to the leads, low temperatures), the cotunneling contribution can be neglected, but it can be essential at larger tunneling couplings and larger temperatures.

Figure 2 shows the dependence of the inverse spin lifetime $\tau_{\sigma}^{-1}$ and the inverse lifetime of the neutral state $\gamma^{\sigma 0}$ on the scaled electron-vibron interaction constant $\sqrt{g}=\lambda / \omega_{0}$. The transition rates from the neutral state to the charged states and vice-versa are suppressed compared to the bare tunneling rate $\Gamma$ if $g$ is large. Thus all states are metastable at low temperatures and zero voltage. Moreover, the lifetime of the charged states can be much larger than that of the neutral state. The last condition is important because then a pure spin-memory effect can be observed without charge memory, see below.

\section{SWITCHING}

In the following we analyze whether fast switching between the two spin states is feasible. To this end we consider

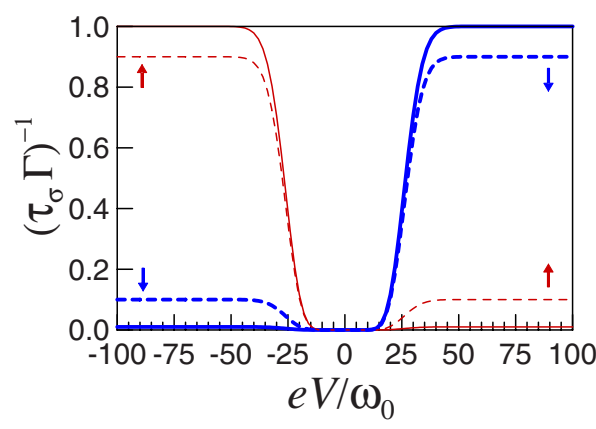

FIG. 3. (Color online) Inverse spin lifetime $\tau_{\sigma}^{-1}=\gamma^{0 \sigma}$ as a function of normalized bias voltage $\mathrm{eV} / \omega_{0}$ at $\lambda / \omega_{0}=3, \epsilon_{0}$ $=\lambda^{2} / 2 \omega_{0}, T=0.1 \omega_{0}$, for the spin-up state (thin red lines) and the spin-down state (thick blue lines) for two different polarizations in the leads: strongly polarized ( $\kappa=0.01$, solid lines) and the same for a less polarized junction $(\kappa=0.1$, dashed lines).

voltage sweeps with different velocities, $\tau_{\text {exp }}$ is the characteristic time of the voltage change. At this point an assumption about the relaxation time $\tau_{V}$ of the vibrons without change in the charge state is due. We assume that the relaxation is fast, $\tau_{V} \ll \tau_{\sigma}, \tau_{\text {exp }}$ so that after an electron tunneling event the system relaxes rapidly into the vibronic ground state $|\sigma 0\rangle$ or $|00\rangle$. In this case the probabilities $P^{\sigma}=\Sigma_{q} P_{q}^{\sigma}$ of the charged states and $P^{0}=\Sigma_{q} P_{q}^{0}$ of the neutral state are determined from

$$
\begin{gathered}
\frac{d P^{0}}{d t}=\sum_{\sigma}\left(\gamma^{0 \sigma} P^{\sigma}-\gamma^{\sigma 0} P^{0}\right), \\
\frac{d P^{\sigma}}{d t}=\gamma^{\sigma 0} P^{0}-\gamma^{0 \sigma} P^{\sigma},
\end{gathered}
$$

where the switching rates $\gamma^{\sigma 0}, \gamma^{0 \sigma}$, at finite voltage are calculated from Eqs. (6), (7), and (13)

$$
\begin{aligned}
\gamma^{\sigma 0}(V)= & \sum_{q} \frac{e^{-g} g^{q}}{q !}\left\{\Gamma_{L \sigma} f^{0}\left[\widetilde{\boldsymbol{\epsilon}}_{\sigma}^{\prime}+\omega_{0} q-(1-\eta) e V\right]\right. \\
& \left.+\Gamma_{R \sigma} f^{0}\left(\widetilde{\boldsymbol{\epsilon}}_{\sigma}^{\prime}+\omega_{0} q+\eta e V\right)\right\}
\end{aligned}
$$

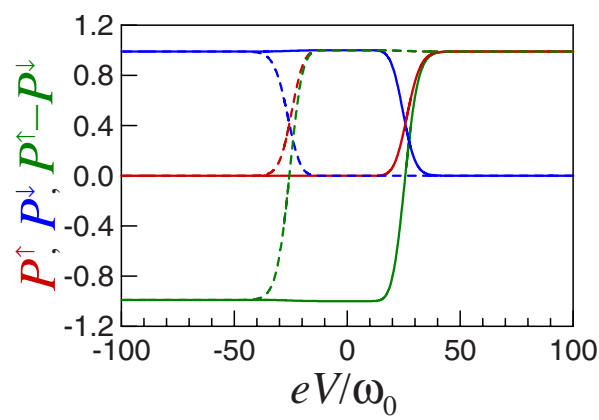

FIG. 4. (Color online) Voltage dependence of the populations of the spin-up state (red), spin-down state (blue), and spin polarization (green) for $\lambda / \omega_{0}=3, \epsilon_{0}=\lambda^{2} / 2 \omega_{0}, \kappa=0.01, T=0.1 \omega_{0}$, solid lines are for increasing voltage and dashed lines for decreasing voltage. 


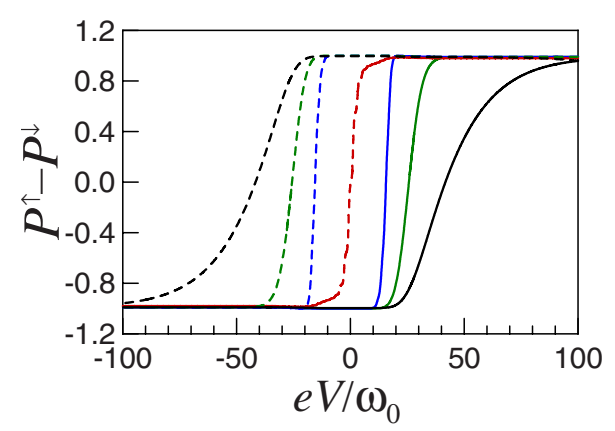

FIG. 5. (Color online) Voltage dependence of the spin polarization for $\lambda / \omega_{0}=3, \epsilon_{0}=\lambda^{2} / 2 \omega_{0}, \kappa=0.01, T=0.1 \omega_{0}$, for three different sweep velocities $\omega_{0} \tau_{\text {exp }}=10^{2}$ (black), $\omega_{0} \tau_{\text {exp }}=10^{3}$ (green), $\omega_{0} \tau_{\text {exp }}=10^{5}$ (blue), solid lines are for increasing voltage and dashed lines for decreasing voltage, the curve in the adiabatic limit $\tau_{\text {exp }} \rightarrow \infty$ is not hysteretic (red dashed line).

$$
\begin{aligned}
\gamma^{0 \sigma}(V)= & \sum_{q} \frac{e^{-g} g^{q}}{q !}\left\{\Gamma_{L \sigma} f^{0}\left[-\widetilde{\epsilon}_{\sigma}^{\prime}+\omega_{0} q+(1-\eta) e V\right]\right. \\
& \left.+\Gamma_{R \sigma} f^{0}\left(-\widetilde{\epsilon}_{\sigma}^{\prime}+\omega_{0} q-\eta e V\right)\right\} .
\end{aligned}
$$

The voltage dependence of the inverse spin lifetimes is depicted in Fig. 3. If the voltage is large enough, the FranckCondon blockade is overcome and the system is switched into the spin-up (spin-down) state at positive (negative) voltage. If the bias voltage is swept fast enough, i.e., faster than the long spin lifetime at zero voltage, $\tau_{\text {exp }} \ll \tau_{\sigma}(0)$, both spin states can be considered as stable at zero voltage (spinmemory effect) and hysteresis can take place. This is shown in Fig. 4 where the solid (dashed) lines mark the spin population for increasing (decreasing) bias voltage. Figure 5 shows how the hysteresis behavior depends on the voltage sweep velocity. In the adiabatic limit the voltage change is so slow that the system relaxes into the equilibrium state, and the population-voltage curve is single-valued, without hysteresis (middle line in Fig. 5). Note that we assume the relaxation of vibrons to be fast. The other time scale-spin lifetime, is actually very long at strong electron-vibron coupling so that small $\tau_{\text {exp }}$ can be large enough in comparison with vibron lifetime.

Finally, we address the question how to discuss the spinmemory effect proposed. It is quite difficult to measure directly a single-spin polarization in an experiment. A reasonable alternative is to study the signatures of the spin polarization in the current-voltage curves which are most easily accessible experimentally. To this end consider now a three-terminal setup, where the third electrode (which can be the gate electrode with tunneling coupling to the system) is ferromagnetic. The tunneling coupling to this electrode is

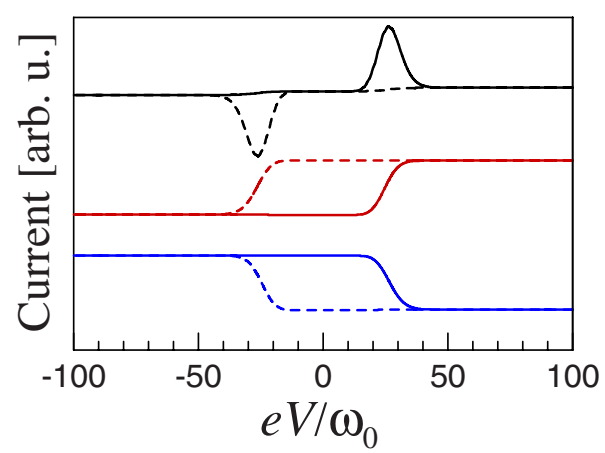

FIG. 6. (Color online) Bias current (top black) and test current for spin-up (middle red) and spin-down (bottom blue) test electrode magnetization as a function of normalized voltage $\mathrm{eV} / \omega_{0}$ for $\lambda / \omega_{0}=3, \epsilon_{0}=\lambda^{2} / 2 \omega_{0}, \quad \kappa=0.01, T=0.1 \omega_{0}$. Solid lines denote increasing voltage and dashed lines decreasing voltage. The curves are displayed with vertical offset in arbitrary units. The magnitude of the test current being much smaller than the bias current, is plotted enhanced.

very weak so that it does not perturb the state. The test tunneling current is calculated simultaneously with the current between left and right electrodes. In Fig. 6 we show the result of this calculation at large negative constant voltage applied to the test electrode. The bias-voltage dependence of the test current is shown as red and blue lines for different orientations of the test electrode magnetization. Since the test current is sensitive to the magnetization of the test electrode, the spin state can be monitored during the experiment. Additionally, such a small current can be used to readout the memory element.

\section{CONCLUSION}

In conclusion, we demonstrated within the framework of a polaron model for a single-level quantum dot coupled to ferromagnetic leads that, owing to Franck-Condon blockade, quantum switching between the two spin states of the central system is strongly suppressed at small bias, but applying a finite bias, the population of spin-up and spin-down states can be controlled. By taking into account nonstationary effects, in particular, the interplay between the time scales of bias sweeping and spin switching, we demonstrate electrically controlled hysteretic behavior and bistability in the spin polarization.

\section{ACKNOWLEDGMENTS}

This work was funded by the Deutsche Forschungsgemeinschaft within the Priority Program SPP 1243 and Collaborative Research Center SFB 689.
${ }^{1}$ C. Iacovita, M. V. Rastei, B. W. Heinrich, T. Brumme, J. Kortus, L. Limot, and J. P. Bucher, Phys. Rev. Lett. 101, 116602 (2008).

${ }^{2}$ F. Meier, L. Zhou, J. Wiebe, and R. Wiesendanger, Science 320, 82 (2008).
${ }^{3}$ X. H. Qiu, G. V. Nazin, and W. Ho, Phys. Rev. Lett. 92, 206102 (2004).

${ }^{4}$ S. W. Wu, G. V. Nazin, X. Chen, X. H. Qiu, and W. Ho, Phys. Rev. Lett. 93, 236802 (2004). 
${ }^{5}$ J. Repp, G. Meyer, S. M. Stojković, A. Gourdon, and C. Joachim, Phys. Rev. Lett. 94, 026803 (2005).

${ }^{6}$ A. C. Hewson and D. M. Newns, J. Phys. C 12, 1665 (1979).

${ }^{7}$ A. S. Alexandrov and A. M. Bratkovsky, Phys. Rev. B 67, 235312 (2003).

${ }^{8}$ M. Galperin, M. A. Ratner, and A. Nitzan, Nano Lett. 5, 125 (2005).

${ }^{9}$ A. Mitra, I. Aleiner, and A. J. Millis, Phys. Rev. Lett. 94, 076404 (2005).

${ }^{10}$ D. Mozyrsky, M. B. Hastings, and I. Martin, Phys. Rev. B 73, 035104 (2006).

${ }^{11}$ D. A. Ryndyk, P. D’Amico, G. Cuniberti, and K. Richter, Phys. Rev. B 78, 085409 (2008).

${ }^{12}$ P. D'Amico, D. A. Ryndyk, G. Cuniberti, and K. Richter, New J. Phys. 10, 085002 (2008).

${ }^{13}$ J. Repp, G. Meyer, F. E. Olsson, and M. Persson, Science 305, 493 (2004).

${ }^{14}$ S. Datta, W. Tian, S. Hong, R. Reifenberger, J. I. Henderson, and C. P. Kubiak, Phys. Rev. Lett. 79, 2530 (1997).
${ }^{15}$ T. Rakshit, G.-C. Liang, A. W. Ghosh, M. Hersam, and S. Datta, Phys. Rev. B 72, 125305 (2005).

${ }^{16}$ I. G. Lang and Y. A. Firsov, Sov. Phys. JETP 16, 1301 (1963).

${ }^{17}$ A. C. Hewson and D. M. Newns, Jpn. J. Appl. Phys., Suppl. 2, 121 (1974).

${ }^{18}$ S. Braig and K. Flensberg, Phys. Rev. B 68, 205324 (2003).

${ }^{19}$ A. Mitra, I. Aleiner, and A. J. Millis, Phys. Rev. B 69, 245302 (2004).

${ }^{20}$ J. Koch and F. von Oppen, Phys. Rev. Lett. 94, 206804 (2005).

${ }^{21}$ J. Koch, M. Semmelhack, F. von Oppen, and A. Nitzan, Phys. Rev. B 73, 155306 (2006).

${ }^{22}$ J. Koch, F. von Oppen, and A. V. Andreev, Phys. Rev. B 74, 205438 (2006).

${ }^{23}$ K. Huang and A. Rhys, Proc. R. Soc. London, Ser. A 204, 406 (1950).

${ }^{24}$ W. Rudziński and J. Barnaś, Phys. Rev. B 64, 085318 (2001).

${ }^{25}$ W. Rudziński, J. Barnaś, R. Świrkowicz, and M. Wilczyński, Phys. Rev. B 71, 205307 (2005). 